Research Article

\title{
Dynamic Evolution of Public's Positive Emotions and Risk Perception for the COVID-19 Pandemic: A Case Study of Hubei Province of China
}

\author{
Yongbao Zhang, ${ }^{1}$ Jingqi Gao, ${ }^{1}$ Xiaowei Luo, ${ }^{2}$ Xiang Wu ${ }^{D},{ }^{1}$ and Haoqi Bao ${ }^{3}$ \\ ${ }^{1}$ School of Engineering and Technology, China University of Geosciences, Beijing 100083, China \\ ${ }^{2}$ College of Engineering, City University of Hong Kong, Hong Kong 999077, China \\ ${ }^{3}$ School of Energy Engineering, Zhejiang University, Hangzhou 310058, China \\ Correspondence should be addressed to Xiang Wu; wuxiang@cugb.edu.cn
}

Received 13 October 2020; Revised 1 January 2021; Accepted 21 March 2021; Published 31 March 2021

Academic Editor: Xander Wang

Copyright ( ) 2021 Yongbao Zhang et al. This is an open access article distributed under the Creative Commons Attribution License, which permits unrestricted use, distribution, and reproduction in any medium, provided the original work is properly cited.

\begin{abstract}
The spread of COVID-19 pandemic and the participation of Internet information are continually changing the public's positive emotions and risk perception. However, relatively little is known about the underlying mechanism of how the COVID-19 dynamic situation affects the public's risk perception and emotions. This study uses the social risk amplification framework (SRAF) as the theoretical basis to collect and analyze Hubei Province data from January 20 to April 8, 2020, including the number of newly diagnosed people per day, the proportion of positive emotional posts in Weibo, and the Baidu search index (BSI). The autoregressive integrated moving average (ARIMAbased time-series prediction model is used to analyze the dynamic evolution laws and fluctuation trends of Weibo positive emotions and risk perception during the development of the pandemic. The conclusion of the study is that positive emotions are negatively correlated with risk perception, the severity of the pandemic situation is negatively correlated with positive emotions, and the severity of the pandemic situation is positively correlated with risk perception. The public has a keen response to the dynamics of the pandemic situation and the government's decision-making behavior, which is manifested by the significant changes in positive emotions and risk perception in the corresponding period. The research results can provide a reference for government departments to guide the public to establish an objective risk perception and maintain positive and stable emotions in similar catastrophes.
\end{abstract}

\section{Introduction}

So far, COVID-19 is still spreading around the world. Almost all countries and regions have adopted different forms and different levels of response measures, whether at the government, organization, or individual level [1]. Articles in the Lancet, an authoritative medical journal, prove that, in the process of responding to the COVID-19 pandemic, personal behavior is essential to control the spread of the virus [2]. The public's emotions and risk perceptions have been shown to significantly affect individual behavior [3], not only changing the normal working lifestyle of the public but also affecting the stability of social order [4].
Risk perception is an activity in which the public uses their intuition to conduct a risk assessment [5]. Human behavior and response depend on the risk they perceive [6]. The public's risk perception of COVID-19 is not only affected by external environments such as economic level, media reports, and government management [7] but also closely related to personal emotions and psychological states [8]. The greater perceived risk in a particular situation usually corresponds to more cautious behavior and more negative emotions [9]. The social risk amplification framework (SRAF) can reflect how emotional stability and prior preparation affect risk perception. It is believed that the interaction of emergencies with psychological, social, and cultural factors may weaken or amplify risk perception [10]. 
Perceived risk is an important driving force for the public to accept the government's epidemic prevention policies and adopt preventive behaviors. Inadequate government preparation and lack of effective supervision will lead to disastrous results [11]. Joel surveyed Twitter users in 12 countries during the epidemic and pointed out that, in crisis situations, social media plays an important role in monitoring public risk perception and guiding public communication [12]. Yan's research on Chinese people shows that, in the early stages of the pandemic, if social media promptly conveys information from the government and decision makers, it can guide people's behavior and minimize the losses caused by the pandemic [13].

In the context of the Internet era, the public's behavior choices in the event of a crisis are susceptible to the risk perception and emotional infection brought about by the large amount of information generated by social media in the short term. During the pandemic, especially in isolation and lockdown, the public's information sharing through social media has attracted more and more attention [12]. The public's understanding of the virus and the risks of the current pandemic are not clear enough, and each country is different. It even shows extreme cases of public overreaction and indifference to the crisis $[14,15]$. Irrational activities during the pandemic are not conducive to effective selfprotection by the public and have a great negative impact on the antipandemic activities of the entire society. To this end, it is important to keep abreast of the public opinion of the COVID-19 pandemic and accurately assess the dynamic evolution of positive emotional and risk perception in crises.

Similar to Twitter, Sina Weibo is the most widely used social networking platform in China and has become a place for gathering information [16]. It contains a large number of news hotspots and the emotional tendency of netizens and plays an important role in the spread of emergencies and other emergencies [17]. Baidu is the most popular search engine in China. Baidu search volume is not the exact search volume of a word, but the weighted sum of the query frequency of the word called Baidu search index (BSI) [18]. It is considered as a reflection of the user's attention to certain content [19]. Due to the huge influence, emergencies often attract the attention of the whole society in a short period, and there will be a sharp increase in related search volume and active comment areas in Weibo, Baidu, and other social media, forming a large number of complex and diverse data. In the past, the research on emotions mainly used questionnaires, and the results were often biased; the research on risk perception was mainly concentrated natural disasters such as typhoons and earthquakes, and the research methods were traditional; the time-series prediction research was mainly applied to the field of economics and finance, and quantitative comparisons of public's emotions were rarely conducted. For the above reasons, this study focuses on Hubei Province, China (one of the regions severely damaged by the pandemic), uses the number of newly diagnosed people every day to reflect the severity of the pandemic, uses Weibo posts to obtain public's positive emotions data, and uses BSI to reflect the degree of risk perception. Based on the analysis by SPSSAU online application software (version
20.0. https://spssau.com), this paper uses autoregressive integrated moving average (ARIMA) time-series prediction model to show the overall trend and abnormal changes and combined current news and Weibo comments for data analysis and interpretation.

In the context of the COVID-19 pandemic and community lockdown, it is of great significance to study the relationship and dynamic trends among the public' positive emotions, risk perception, and severity of the pandemic in Hubei Province. The data collection and data analysis methods of this study are novel and reasonable, and the results are quite convincing, which can provide references for similar disaster research. This research can scientifically and effectively analyze the positive emotions and risk perceptions of the public in Hubei Province in response to COVID-19 and provide theoretical basis and suggestions for government departments to guide the public to establish correct risk perceptions and maintain stable emotions during disasters.

\section{Literature Review}

2.1. Positive Emotions. Fredrickson believes that positive emotions are a person's unique response to things, and it is a temporary pleasure [20]. According to the theory of emotion cognition, emotions are given or set by human beings in the process of evolution to adapt to the environment [21]. Both positive and negative emotions have evolved gradually in response to an environment with existential threats and are closely related to certain events. Traditional emotion analysis technology is mainly based on natural language processing and machine learning. It determines the emotional orientation of the text by analyzing the relationship between emotional expression and the topic or using data to test and train the machine [22]. These methods require a lot of data and complex operations, so emotional research with the help of the Internet has gradually shown its own advantages.

With the rapid development of social media, social networks such as Twitter, Weibo, and Facebook are gradually changing people's lives. More and more people are willing to express their attitudes and emotions on social networks. Therefore, more and more researchers are applying the emotional theory of psychology to the emotional analysis of online texts. A nine-year study shows that Internet behavior can predict people's positive emotions [23]. On the basis of the Profile of Mood States (POMS) psychological scale, Pepe and Bollen constructed the POMS-1 scale suitable for Twitter research, which can measure the public's mental state from six aspects. They asked subjects to send e-mails to their future selves and analyzed subjects' attitudes towards the future from the emotions contained in the e-mails. Pepe and Bollen matched the vocabulary to the emotion words in the e-mail, and one point would be added when an emotional word appeared. A numerical value is obtained after accumulation, and the corresponding emotional index is obtained by standardization and other processing [24].

For the classification of emotions, Dong et al. used the relevant theory of emotional structure to create a basic social 
emotion measurement vocabulary based in Weibo and divided basic social emotions into five categories: happiness, disgust, sadness, anger, and fear [25]. Watson and Tellegen summarized a "positive-negative emotion model," that is, positive and negative emotions are two basic dimensions independent of each other [26]. For the measurement of emotion, Kim chose vocabulary matching technology to define the emotional tendency of the text by judging whether there was a vocabulary in the lexicon. Jiang et al. studied the expression and measurement of emotions in Weibo emoticons [27]. During the COVID-19 pandemic, the people of Hubei are under lockdown. The long-term community closure affects the public's mental health [28]. However, there are few studies on public positive emotions in public health emergencies; thus, further research and analysis are needed. Based on the above, this study uses Internet tools to collect positive emotion data in Weibo during the COVID19 pandemic, which can more scientifically and effectively analyze the dynamic changes of the public's positive emotion in response to crisis events.

2.2. Risk Perception. Risk perception is a process in which the public judges the dangers of the external environment and makes relevant decisions based on their own subjective feelings about risks [5]. Research on risk perception originated from psychological research. Since then, due to frequent natural disasters and man-made accidents, risk perception and risk management research have become more closely linked. Starr found that people's perception of risk not only is related to the evaluation of risk itself but also takes into account people's subjective scales [29]. Fischhoff and Slovic obtained the risk preference and the trade-off standard between risk and return through questionnaire surveys and proposed a psychometric paradigm in the field of risk management [30], which greatly promoted and developed the research progress of risk perception.

Slovic et al. [31] believed that risk perception could be quantified and predicted through five levels, namely, frequency of death, subjective fatal estimation, potential for disasters, significance of death, and qualitative characteristics. Thirty years ago, Weinstein and Douglas explored the public's risk perception of disaster events from the perspective of social-cultural theory paradigm and believed that the individual's social background, cultural level, belief, and other factors are closely related to public risk perception [32]. Recent studies have shown that people's perception of pandemic risk is influenced by factors such as personality traits, gender, media, economy, and government [33]. Generally speaking, people often rely on the news media as a source of information exchange to assess risks. Therefore, the media will influence their initial understanding of the disease. At the same time, values and culture are quietly affecting the pandemic prevention behaviors of different countries and regions [34]. Bronfman and Cifuentes used the individual roles, human benefits, and people's fear of risk as measurement factors to explore the differences in risk perception among different groups [35]. Roberts explored the impact of panic on people's risk perception by building- related models [36]. Coyne studied the psychological effects of the combined effect of the subjective and objective emotions of the affected population under stress. It is believed that the postdisaster emergency response emotion is the main factor causing the psychological fluctuations of the affected people [37]. Recent studies have shown that the attention and emotional expression of the epidemic information in Twitter can be used to describe the public's risk perception of COVID-19 [12]. Tang analyzed and calculated the public's perception of social risks based on the Baidu Search Index (BSI) [38]. In order to quantitatively study the public's risk perception of COVID-19, we applied this method to express the risk perception with the frequency of online searches.

In summary, most current research studies believe that risk perception is one of the important driving factors of behavioral decision-making, and the influence of the emotions of disaster-affected groups on risk perception and behavior has also become the research direction of some scholars. In the context of such a serious global pandemic, a comprehensive exploration of public' risk perceptions and emotions has more theoretical and practical significance.

\subsection{Social Risk Amplification Framework (SRAF).} Kasperson first puts the effect of risk communication under the social background to conduct research, revealing the nature of risk and quantifying its level from the perspective of information dissemination network and public response. He proposed that, in the process of communication from the source of information to the dissemination intermediary and then to the recipient of the information, the signal would increase or decrease, and the information content would also be distorted to a certain extent. This phenomenon is defined as the social amplification effect of risk in the field of risk communication and is also called as the social risk amplification framework (SRAF). SRAF can help to explain the two stages of information. The first stage focuses on transmitting the information to the amplification station, conducting social awareness understanding and impact assessment, and then taking action. The second stage is the reaction and influence mechanism within the society [39].

In real life, the SRAF of risk perception usually leads to adverse consequences. It can enlarge or reduce the unknown risks and potential threats caused by accidents, pollution, and outbreaks. The impact of the amplification process of risk perception sometimes exceeds the direct impact of the disaster itself. The SRAF has an important impact on the dissemination of disaster warning information at the level of emergency evacuation strategies and, therefore, has become a major research focus in the current risk management field. Through the study of dangerous information flow and risk perception, Lindell and Perry built a decision-making model of individual protection behavior and identified the threestage decision-making process of public protection behavior [40]. In addition, they also analyzed the family structure of people in multihazard areas and explored the internal relationship among it, individual risk perception, and protective behavior decision-making [41]. 
The SRAF can reflect how emotional stability and preparation affect risk perception. It is believed that the interaction of emergencies and psychological, social, cultural, and other factors may weaken or amplify risk perception. If the risk perception is amplified, it indicates that the individual has lower emotional stability, which is characterized by anxiety, aggression, and pessimism; on the contrary, the decrease of risk perception shows higher characteristics of emotional stability, such as optimism and calm. At the same time, emotional stability and prepreparation are complementary. Higher emotional stability enables individuals to prepare and respond to emergencies more fully [10] and respond positively and optimistically to disasters.

2.4. ARIMA Time-Series Analysis. ARIMA time-series analysis is a method for processing dynamic data in chronological order based on the recorded data. It relies on curve fitting and parameter estimation to analyze its trend over time, investigate its own statistical laws, and then predict the future value of the target [42]. The ARIMA time-series prediction model is also denoted as $\operatorname{ARIMA}(p, d, q)$. The $\operatorname{ARIMA}(p, d, q)$ model is a summed autoregressive moving average model, where $p$ is the number of autoregressive terms, $q$ is the number of moving average terms, and $d$ is the number of differences made to make the original sequence stationary. To find the best model prediction result, it is necessary to use the principle of minimum Akaike information criterion (AIC) value, through statistical analysis, to obtain the optimal prediction of the future value of the sequence.

Time-series analysis has been widely used in finance, climate change, industrial production, and other fields. $\mathrm{Li}$ et al. performed time-series analysis and modeling of aerosols between the United States and China from 2003 to 2015. The results showed that ARIMA had a good prediction ability for time-series with significant seasonality [43]. Perzyk et al. took the product temperature in the factory as an example to conduct time-series prediction analysis and detect and quantitatively evaluate the overall trend, periodicity, and amplitude of parameter changes [44]. Narayanan et al. used ARIMA time-series analysis to study the trend of premonsoon precipitation in western India [45]. Singh et al. used the death toll data of five countries with severe COVID-19 outbreaks to establish a comprehensive prediction model based on ARIMA time series [46]. Coincident with this article, we all hope that the forecast results can help the government take countermeasures in advance.

\section{Method}

This section gives a detailed introduction to the sources of the research data and analysis methods. First, select the research period and object according to the objective reality and obtain the original data from Weibo, Baidu, official website of the National Health Commission of China, and other platforms, respectively, and then classify the emotion of Weibo to select posts with public's positive emotions.
Then, the three variables, the proportion of positive emotional posts, the BSI, and the daily increase in the number of diagnoses, were analyzed by correlation and ARIMA timeseries prediction. Under the SRAF framework, the dynamic changes of the public's positive emotions and risk perception during the global pandemic are explored.

3.1. Research Subjects and Research Period. This study selects Weibo users in Hubei Province, China, as the analysis subjects, for the following reasons. First, within the territory of China, COVID-19 was first discovered in the South China Seafood Wholesale Market in Wuhan, Hubei. Second, Hubei Province is the hardest hit by the COVID-19 pandemic in China. The Hubei Weibo data collected in this study take January 20, 2020, as the starting time. From that day on, the National Health Commission of China began to publish the national (province) pandemic data, taking April 8, 2020, as the deadline, and on that day, Wuhan City, Hubei Province, announced the lifting of the city blockade.

\subsection{Data Collection}

3.2.1. The Proportion of Positive Emotional Posts in Weibo. This research used the advanced search function of Sina Weibo (version 10.6.2) to search for all original posts with the keyword “COVID-19" from January 20, 2020, to April 8, 2020, in Hubei area in units of days, and 20 samples were taken evenly every day using systematic sampling techniques. The total samples include 1600 Weibo posts for 80 days. Take January 20, 2020, as an example to introduce the Weibo sampling process. Firstly, we set the time period from 0:00 on January 20, 2020, to 0:00 on January 21, 2020, entered the keyword "COVID-19," the limited area is Hubei, the post type is original, and the search results in a total of 974 Weibo posts, $N=947$, and numbered from top to bottom. Secondly, in order to ensure that the daily sample is representative, 20 posts were selected at regular intervals from 947 Weibo posts, $n=20$. According to the formula $k=N / n$, the number of intervals for extracting Weibo is calculated, and the interval $k=49(974 / 20=48.7$, take an integer). Finally, the extraction is performed at an interval of 49, and finally, 20 Weibo posts are extracted.

Refer to Dong's microblog-based basic social emotion measurement vocabulary for emotion classification, which divides 818 words into five basic social emotions: happiness, sadness, disgust, anger, and fear [25]. According to the positive-negative emotion model proposed by Watson and Tellegen, the above five basic social emotions are divided into positive and negative emotions.

Emotional classification of extracted Weibo posts: if happy emotions such as "Happy" and "Relief" are found in the post, we add 1 to the value of positive emotions (statistics in positive emotions); if we find words such as "sarcasm," "confused," and "panic," we add 1 to the value of negative emotions (statistics in negative emotions) [16]. Finally, the positive and negative Weibo values are accumulated, and the proportion of positive emotional posts in Weibo is calculated. What needs to be emphasized is that when dividing 
emotions if the following two posts that are not suitable for cooperation in this research are statistically required, then select nearby posts as a substitute. The principle of substitution is to give priority to the data below the invalid Weibo and then consider the above, until the Weibo posts of a certain emotion is judged. The details are as follows:

(1) If there are no obvious emotion words or multiple emotion words in the Weibo posts, which cannot determine the specific emotion of the Weibo posts, then select other nearby Weibo posts instead

(2) Since this study mainly discusses the public's views on COVID-19, the Weibo posts published by newspapers, media, government, and other subjects are not the public's emotional expression, so they are not included in the statistics, and other Weibo posts in the vicinity will be selected instead

3.2.2. Baidu Search Index (BSI). Baidu News hot word search engine platform provides daily BSI. This study draws on the frequency of online search behavior proposed by Tang and $\mathrm{Xu}$ to express risk perception $[19,38]$ and uses Baidu News hot word search engine to retrieve the keyword "COVID-19."

The search time is from January 20 to April 8, 2020, and the tool is Baidu News hot word search engine "PC terminal + mobile terminal" (http://index.baidu.com). By using the search volume of Hubei Province netizens on Baidu website as the database and COVID-19 as the object, scientifically analyze and calculate the frequency of the COVID-19 search in Baidu web search, weighted and standardized to obtain BSI, which can be used to express the level of daily public's risk perception.

\subsubsection{The Daily Increase in the Number of Diagnoses.} The number of newly confirmed cases is a key factor in measuring the severity of the pandemic [47]. This study counted the newly diagnosed people every day in Hubei Province published by the official website of the National Health Commission of China (http://www.nhc.gov.cn) from January 20 to April 8, 2020. The number of newly diagnosed people every day is used to reflect the severity of the COVID19 pandemic.

3.2.4. Data Summary. After 80 days of statistics and sorting, the original data of the proportion of positive emotional posts in Weibo, the Baidu Search Index (BSI), and the number of newly diagnosed people per day are formed. The original data are the basis for subsequent analysis and drawing. However, it is not difficult to find from the original data that the largest value is BSI, which is 83928 , and the smallest value is the proportion of positive emotions, which is $20 \%$. The range of data on the proportion of positive emotional posts, the BSI, and the number of newly diagnosed persons per day is very large. In order to better horizontal comparison and linear regression analysis, the data were standardized by $z$-score.
For example, when displaying the trend of three variables in a graph or performing linear regression analysis, the standardized data are used. The principle of the $z$-score standardization method is to rely on the standard deviation and the mean of the original data for standardization. It is the most common standardization method by default in SPSSAU online statistical analysis software (version 20.0, https://spssau.com). The conversion result retains four decimal places. After standardization, the proportion of positive emotional posts in Weibo is used to reflect the public's positive emotions, the BSI is used to reflect the risk perception level, and the number of newly diagnosed people is used to reflect the severity of the pandemic.

\subsection{Data Analysis Methods}

3.3.1. Correlation Analysis. In this study, SPSSAU online analysis software (https://spssau.com) was used to conduct a correlation analysis. The analysis factor is the raw data of the three sets of variables: the proportion of positive emotional posts, the BSI, and the number of newly diagnosed people every day.

3.3.2. Linear Regression Analysis. Linear regression analysis is a method of predicting the future value of a random variable related to it based on the change of one or a group of independent variables. Regression analysis needs to establish a regression equation describing the correlation between variables. If the regression function is a linear function, it is said that the variables are linearly related. This three-group linear analysis is one-variable linear regression analysis, including two variables, one is the independent variable and the other is the dependent variable. When performing the linear regression analysis, it is more appropriate to use standardized data.

In the $F$ test method of linear regression analysis, the adjusted $R^{2}$ is used to indicate the degree of explanation of the independent variable to the dependent variable; the standardized coefficient indicates the coefficient of the independent variable in the regression equation, and the larger the value, the greater the impact on the independent variable 'Larger'. The $F$ value is the result of analysis of variance, which is used to summarize the significance of the regression equation. The $p$ value corresponding to the $F$ value is less than 0.05 , and it is considered that there is a correlation between the two variables; the closer the value is to 0 , the better the correlation is.

\subsubsection{ARIMA-Based Time-Series Prediction Model.} ARIMA is a general time-series prediction model. As a data mining tool, time series helps researchers to better understand the nature of the development process of things and can predict the future value of variables based on historical data recorded in chronological order [48].

During the COVID-19 pandemic, public's positive emotions and risk perception will fluctuate greatly as the pandemic develops. In order to explain and predict this kind 
of fluctuations, this study introduces the ARIMA time-series prediction model to visually show the changing laws and fluctuation trends of positive emotions and risk perception. In the model, the fitted value in a different period is compared with the real value, and the objective reasons that affect its change are analyzed according to the objective event.

\subsubsection{Explanatory Analysis of Emotional and Risk Perception} of Online News/Weibo Viewpoints of Obviously Changing Nodes. The explanatory analysis focuses on the positive emotions in Weibo and BSI during the pandemic. On the basis of ARIMA time-series prediction results, in order to further explore the reasons for the significant changes in positive emotions and risk perception, we conducted an explanatory analysis of the rear performance and fitting results of these two variables.

In view of the four objective situations of low positive emotions, high positive emotions, high degree of risk perception, and low degree of risk, we calculated and selected the time periods with the largest deviation between the true value and the fitted value. Then, we collected major news events and pandemic dynamics that may affect emotions and risk perception in this period and explored whether these news events and pandemic dynamics can significantly affect the changes in emotion and risk perception.

\section{Results}

This section uses the research methods provided above to organize and analyze the data. Firstly, we used SPSSAU to perform basic analysis such as standardization and normality test on the data, then performed correlation and linear regression analysis, and drew the change trend graph of the three sets of variables. In addition, the ARIMA timeseries prediction model is used to fit and predict the proportion of positive emotional posts in Weibo and BSI. Finally, we combined major news events and pandemic dynamics during the pandemic to explain and analyze the actual causes of significant fluctuations in public emotions and risk perception.

4.1. Normality Test. Perform normality test analysis on the number of newly confirmed cases, the BSI, and the proportion of positive emotional posts. Because the samples of the three sets of data are larger than 50 samples, the $K-S$ test method is selected. In detail, the number of newly confirmed cases, the Baidu search index, and the proportion of positive emotion microblogs all show significant $(p<0.05)$, which means the number of newly confirmed cases, the BSI, and the proportion of positive emotional posts do not have the characteristics of normality as shown in Table 1 .

4.2. Correlation Analysis of the Number of Newly Confirmed Cases, the BSI, and the Proportion of Positive Emotional Posts. Through the preliminary analysis of SPSSAU, it is found that the three sets of data in this study do not have the characteristic of normality, so the Spearman correlation coefficient is used to indicate the strength of the correlation. The results of the correlation analysis are shown in Table 2.

The correlation analysis results show that the three sets of data are significant at the 0.01 level, which proves that there is a strong correlation between the number of newly confirmed cases, the BSI, and the proportion of positive emotional posts.

\subsection{Linear Regression Analysis}

4.3.1. Linear Regression Analysis of Positive Emotion and Risk Perception. According to the correlation analysis results and literature data, we take the standardized proportion of positive emotions as the independent variable and the standardized BSI, namely, risk perception, as the dependent variable and perform linear regression analysis. The analysis results are shown in Table 3.

Linear regression analysis found that the $R^{2}$ value of the model is 0.651 , which means that the proportion of positive emotional posts in Weibo can explain the $65.1 \%$ change in risk perception. The model passed the $F$ test $(F=145.576$ and $p \leq 0.001)$, which means that the proportion of positive emotions will affect risk perception. The model formula is BSI $=0.000-0.807^{*}$ proportion of positive emotions.

The regression coefficient value of the proportion of positive emotional posts in Weibo is -0.807 ( $t=-12.065$ and $p \leq 0.001$ ), which can be summarized as the proportion of positive emotional posts in Weibo has a significant negative correlation with risk perception.

4.3.2. Linear Regression Analysis of the Severity of the Pandemic Situation and the Proportion of Positive Emotional Posts. Using the same method as the above linear regression analysis, the number of newly diagnosed cases after standardization, that is, the severity of the pandemic was used as the independent variable, and the proportion of positive emotional posts in Weibo was the dependent variable. Linear regression analysis was performed. The $R^{2}$ value of the model is 0.228 , which means that the severity of the pandemic can explain the reason for the change of $22.8 \%$ of the proportion of positive emotions. The model passed the $F$ test $(F=22.984$ and $p \leq 0.001)$, which means that the severity of the pandemic will affect the proportion of positive emotional posts in Weibo. The model formula is the proportion of the positive emotional posts $=0.000-0.477 *$ the number of newly diagnosed people.

The regression coefficient of the severity of the pandemic is $-0.477(t=-4.794, p \leq 0.001)$, which can be summarized as follows: the severity of the pandemic has a significant negative correlation with the proportion of the positive emotional posts in Weibo.

4.3.3. Linear Regression Analysis of the Severity of Pandemic and Risk Perception. Using the same method as the above linear regression analysis, the number of newly confirmed cases after standardization, that is, the severity of the 
TABLE 1: The results of normality test.

\begin{tabular}{|c|c|c|c|c|c|c|c|c|c|}
\hline & \multirow{2}{*}{ Sample } & \multirow{2}{*}{ Mean } & \multirow{2}{*}{ Std } & \multirow{2}{*}{ Skew } & \multirow{2}{*}{ Kurtosis } & \multicolumn{2}{|c|}{$K-S$} & \multicolumn{2}{|c|}{$S-W$} \\
\hline & & & & & & Statistic & $p$ & Statistic & $p$ \\
\hline The number of newly confirmed cases & 80 & 860.638 & 1877.408 & 5.543 & 39.274 & 0.323 & $0.000^{* *}$ & 0.458 & $0.000^{* *}$ \\
\hline BSI & 80 & 26560.63 & 17845.89 & 1.206 & 1.03 & 0.184 & $0.000^{* *}$ & 0.862 & $0.000^{* *}$ \\
\hline The proportion of positive emotional posts & 80 & 0.527 & 0.231 & 0.081 & -1.478 & 0.156 & $0.000^{* *}$ & 0.901 & $0.000^{* *}$ \\
\hline
\end{tabular}

${ }^{*} p<0.05$ and ${ }^{* *} p<0.01$.

TABle 2: The Spearman correlation analysis results.

\begin{tabular}{lccc}
\hline & The number of newly confirmed cases & BSI & The proportion of positive emotional posts \\
\hline The number of newly confirmed cases & 1 & - & - \\
BSI & $0.870^{* *}$ & 1 & - \\
The proportion of positive emotional posts & $-0.869^{* *}$ & $-0.908^{* *}$ & 1 \\
\hline
\end{tabular}

Note. ${ }^{*} p<0.05$ and $^{* *} p<0.01$.

TABLE 3: Linear regression analysis results of the perception positive emotional posts and risk perception.

\begin{tabular}{|c|c|c|c|c|c|c|c|c|c|}
\hline \multicolumn{10}{|c|}{ Linear regression analysis results $(n=64)$} \\
\hline & \multicolumn{2}{|c|}{$\begin{array}{l}\text { Unstandardized } \\
\text { coefficient }\end{array}$} & \multirow{2}{*}{$\begin{array}{c}\text { Standardized } \\
\text { coefficient } \\
\text { Beta }\end{array}$} & \multirow{2}{*}{$t$} & \multirow{2}{*}{$p$} & \multirow{2}{*}{ VIF } & \multirow{2}{*}{$R^{2}$} & \multirow{2}{*}{$\begin{array}{l}\text { Adjust } \\
R^{2}\end{array}$} & \multirow{2}{*}{$F$} \\
\hline & $B$ & $\begin{array}{l}\text { Standard } \\
\text { deviation }\end{array}$ & & & & & & & \\
\hline \multirow{2}{*}{$\begin{array}{l}\text { Constant } \\
\text { The proportion of positive } \\
\text { emotional posts }\end{array}$} & 0.000 & 0.066 & - & 0.000 & 1.000 & - & \multirow[b]{2}{*}{0.651} & \multirow[b]{2}{*}{0.647} & \multirow{2}{*}{$\begin{array}{c}F(1,78)=145.576 \\
p \leq 0.001\end{array}$} \\
\hline & -0.807 & 0.067 & -0.807 & -12.065 & $\leq 0.000^{* *}$ & 1.000 & & & \\
\hline
\end{tabular}

Note. Dependent variable: BSI; D-W value: $0.549 .{ }^{*} p<0.05$ and ${ }^{* *} p<0.01$.

pandemic was used as the independent variable, and the standardized BSI, that is, risk perception, as the dependent variable, was used for linear regression analysis. The $R^{2}$ value of the model is 0.123 , which means that the severity of the pandemic can explain the $12.3 \%$ change in risk perception. The model passed the $F$ test $(F=10.926$ and $p \leq 0.001)$, which means that the severity of the pandemic will affect the risk perception. The model formula is risk perception $=-0.000+$ $0.351 *$ the severity of the pandemic.

The regression coefficient of the severity of the pandemic is $0.351(t=3.305$ and $p \leq 0.001)$, which can be summarized as the severity of the pandemic has a significant positive correlation with risk perception.

\subsection{The Overall Trend of the Proportion of Positive Emotional} Posts, the Number of Newly Diagnosed People, and the BSI. Use SPSSAU to process the standardized proportion of positive emotional posts, the number of newly diagnosed people, and the BSI and draw the changing trend, as shown in Figure 1.

As can be seen in figure 1, the relationship between the three variables generally conforms to the laws derived from the above study, that is, the proportion of positive emotional posts has a significant negative correlation with risk perception, the severity of the pandemic has a significant negative correlation with the proportion of positive emotional posts, and the severity of the pandemic has a significant positive correlation with risk perception.
However, there are abnormal fluctuations in certain periods, indicating a sudden change in public's emotions and risk perception. The reasons for these mutations will be explained objectively below.

4.5. ARIMA Time-Series Prediction Results. In order to explore the deep-seated reasons that affect emotion and risk perception, this paper carries out ARIMA time-series analysis. The model used to predict the proportion of positive emotional posts in Weibo, also the fitting value of BSI, and calculate the deviation of the real value from the fitting value to reflect the fluctuation degree of emotion and risk perception. Figures 2 and 3 are plotted based on the data of the real and fitted values.

4.6. Explanatory Analysis of Emotional and Risk Perception of Online News/Weibo Viewpoints of Obviously Changing Nodes. This section focuses on the proportion of positive emotional posts in Weibo and the BSI during the pandemic. In order to perform an explanatory analysis on the real performance and the fitted results, the point when the real value and the fitted value were significantly different was found, and major news events and Weibo posts that may affect emotion and risk perception were also collected at the time.

4.6.1. Explanatory Analysis of Positive Emotional Fluctuations. (1) Low Positive Emotions. According to the result of prediction and analysis in figure 2 by ARIMA, 


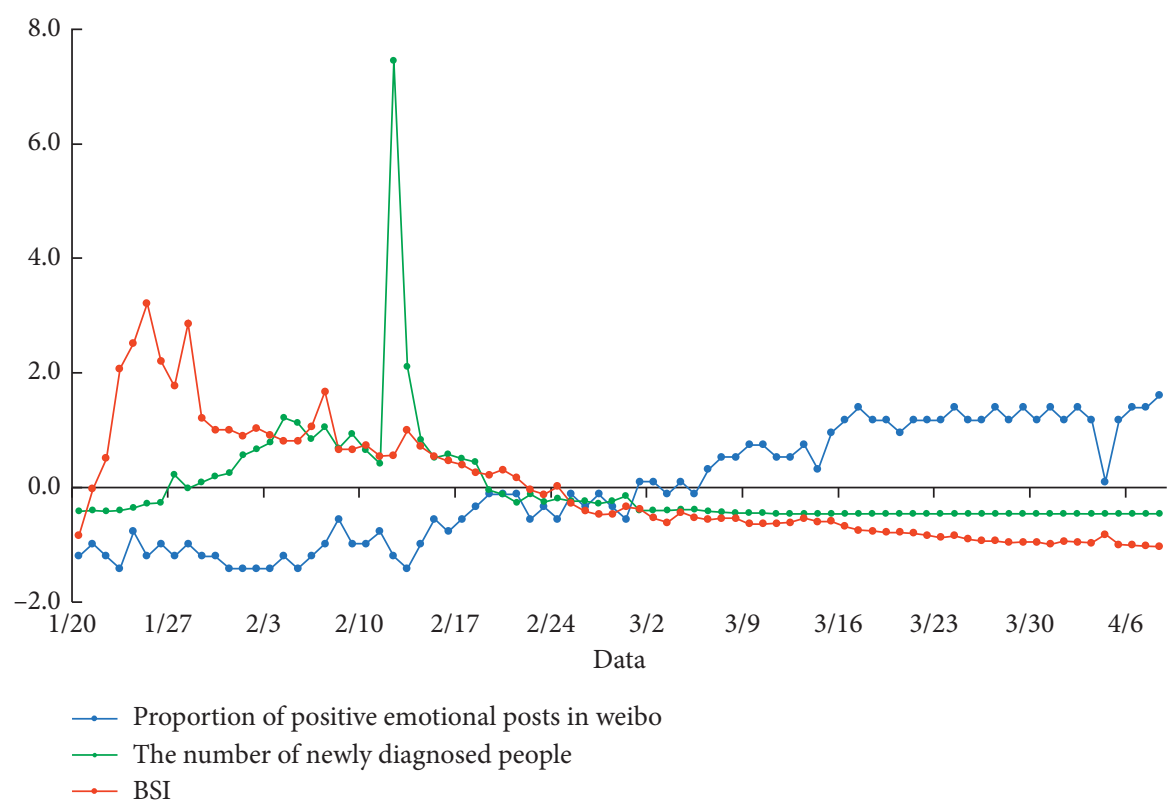

FIGURE 1: Change trend of the proportion of positive emotional posts, the number of newly diagnosed people, and the BSI.

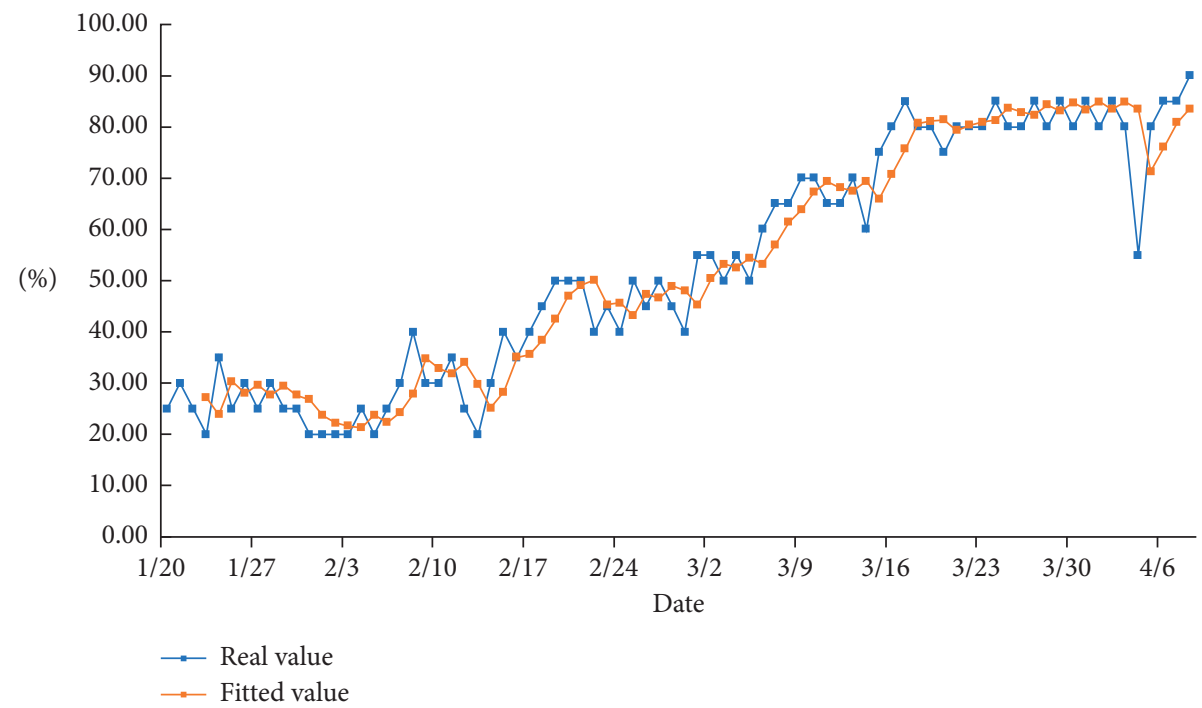

FIGURE 2: Simulated fitting trend graph of the proportion of positive emotional posts.

we selected five days in which the real value of the positive emotions in the Weibo posts was most significantly lower than the fitted value, which is collated in Table 4.

Searching and sorting out the pandemic situation and major news events at the corresponding time points, we found several events as described below. Wuhan blocked the city on January 23; the number of newly diagnosed people per day surged on February 12; many doctors such as Peng Yinhua and Liu Zhiming died on February 22 due to COVID-19; the WHO raised the pandemic risk level on February 29; National Mourning Day was observed on April 4. News events and pandemic dynamics are associated with fluctuations in positive emotions. From the news events shown in Table 4 and above, the real value of the proportion of positive emotional posts is significantly lower than the fitted value, and the public's emotions show a more negative trend, in line with the above analysis. The surge in the number of infected people during the pandemic and the news of the spread of the pandemic will cause the public to panic, the published Weibo views become pessimistic, and the real value of the positive emotion ratio is significantly lower than the fitted value.

(2) High Positive Emotions. According to the result of prediction and analysis in figure 2 by ARIMA, we selected five days in which the real value of the positive emotion in Weibo posts was most significantly higher than the fitted value, which is collated in Table 5. 


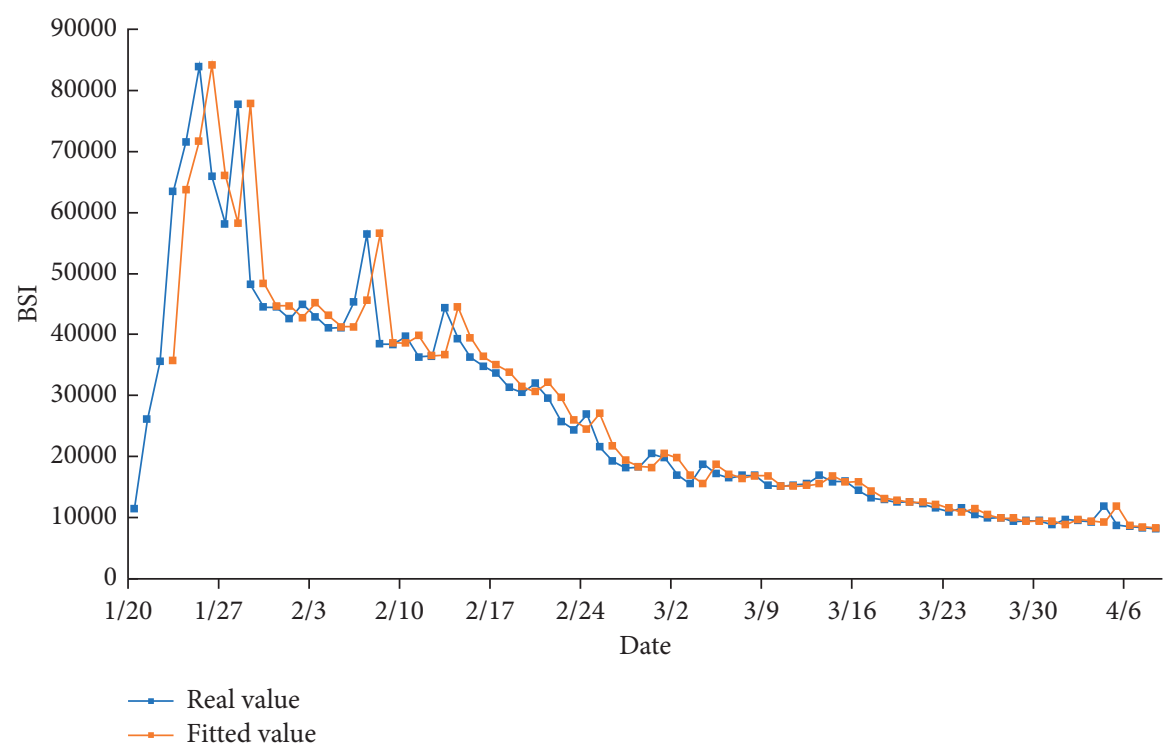

FIGURE 3: Simulated fitting trend graph of BSI.

Searching and sorting out the pandemic situation and major news events at the corresponding time points, we found the Raytheon Mountain Hospital was put into use on February 8; the potential drug "Favipiravir" was approved for listing on February 14-15; the major square cabin hospitals were closed on March 6-10; on March 16-18, there were no newly diagnosed cases in Wuhan; Wuhan lifted the city blockade on April 8. News events and pandemic dynamics are associated with fluctuations in positive emotions. Every positive action and research progress of the government and scientific research institutions, as well as the news events of the weakened pandemic, will make the public more optimistic and publish more positive opinions in Weibo. As shown in Table 5, the real value of the positive Weibo ratio is significantly higher than the fitted value, and the public's emotions show a significantly positive state, which is consistent with the analysis of the objective facts above. It can be concluded from this that news reports will have a great influence on the fluctuation of public's emotion, and the positive information disseminated by the government and the media will play a vital role in stabilizing public's emotion.

\subsubsection{Explanatory Analysis of BSI}

(1) High BSI. According to the result of prediction and analysis in ure 3 by ARIMA, we selected three days in which the real value in BSI is most significantly lower than the fitted value, which is collated in Table 6.

Searching and sorting out the pandemic situation and major news events at the corresponding time points, we found Wuhan blocked the city on January 23; the WHO raised the pandemic risk level on February 29; and National Mourning Day was observed on April 4. The occurrence of the above incidents has caused extensive searches by netizens, and people's perception of risk has been significantly improved. Comparing the data of the proportion of positive
TABLe 4: Period data with a low proportion of positive emotional posts.

\begin{tabular}{lcccc}
\hline Data & Real value (\%) & Fitted value (\%) & Deviation & Symbol \\
\hline $1 / 23$ & 20.00 & 27.27 & 36.33 & - \\
$2 / 12$ & 25.00 & 34.07 & 36.27 & - \\
$2 / 22$ & 40.00 & 50.14 & 25.34 & - \\
$2 / 29$ & 40.00 & 48.10 & 20.26 & - \\
$4 / 4$ & 55.00 & 83.47 & 51.76 & - \\
\hline
\end{tabular}

Note. (1) Deviation = |real value-fitted value //real value [49], which is used to reflect the degree of difference between the real value and the fitted value and to find out the period when the abnormal value occurs. (2) The symbol reflects the relationship between the real value and the fitted value. If the real value is greater than the fitted value, the column of the symbol is "+;" otherwise, it is "-." (3) The percentages here are kept to two decimal places. (4) Tables 5-7 are the same as Table 4.

TABle 5: Period data with a high proportion of positive emotional posts.

\begin{tabular}{lcccc}
\hline Data & Real value $(\%)$ & Fitted value $(\%)$ & Deviation & Symbol \\
\hline $2 / 8$ & 40.00 & 27.86 & 30.35 & + \\
$2 / 14$ & 30.00 & 25.13 & 16.23 & + \\
$3 / 6$ & 60.00 & 53.20 & 11.33 & + \\
$3 / 16$ & 80.00 & 70.80 & 11.50 & + \\
$4 / 8$ & 90.00 & 83.51 & 7.21 & + \\
\hline
\end{tabular}

TABLe 6: Data with high BSI period.

\begin{tabular}{lcccc}
\hline Data & Real value & Fitted value & Deviation & Symbol \\
\hline $1 / 23$ & 63502 & 35747 & 43.71 & + \\
$2 / 29$ & 20507 & 18235 & 11.08 & + \\
$4 / 4$ & 11943 & 9252 & 22.53 & + \\
\hline
\end{tabular}

emotional posts in Weibo in the same period, the real value of the three-day data is significantly lower than the fitted value, and the emotion tends to be negative. This finding also validates the research conclusion of Bhandari and Hallowell 
[50], namely, positive emotions will make the public underestimate the perceived risks, negative emotions will increase people's risk perception, and the proportion of positive emotional posts has a negative correlation with risk perception.

(2) Low BSI. According to the result of prediction and analysis in ure 3 by ARIMA, we selected three days in which the real value in BSI is most significantly higher than the fitted value, which is collated in Table 7 .

Searching and sorting out the pandemic situation and major news events at the corresponding time points, we found Raytheon Mountain Hospital was put into use on February $8^{\text {th }}$; IgM/IgG antibody rapid test kit was put into use on February 14-15, and the potential drug "Favipiravir" was approved for listing on the same day; on March $16^{\text {th }}$ to $18^{\text {th }}$, the first batch of Chinese National Aid Medical Team returned, and there were no newly diagnosed people in Wuhan. The government's positive measures and scientific research progress have reduced people's perception of risk, and the frequency of online searches has been reduced. It can be seen from Table 7 that the corresponding BSI has decreased over the past few days, which is lower than the fitted value. Besides, these days are also days when the proportion of positive emotional posts in Weibo is high, and people's emotions are very positive. From this, we can draw a conclusion similar to Choi [51] and Duan et al. [52]. Government actions and authoritative media reports will affect people's perception of risk. Accordingly, the BSI will change greatly. The government and authoritative media will play a critical role in reducing the public's perception of risk.

\section{Discussion}

Although the research on emotion or risk perception has been quite in-depth in many fields, we believe that, in the context of such a severe COVID-19 pandemic, it is still relatively novel and of great significance to comprehensively analyze the public's positive emotions and risk perception. Hubei, as the region with the worst pandemic in China, has basically returned to normal living order. However, the world is still in the battle for a long time with COVID-19 [53]. The pandemic information on the Internet and reality will affect the public's emotions and risk perceptions, which in turn will affect individuals and even social behavior. Therefore, based on network data, this article studies the public's positive emotions and risk perceptions during the pandemic situation and supplements and improves the viewpoints of previous studies, to provide a useful reference for the analysis of public psychology and behavior under such sudden disasters.

5.1. Discussion of Research Methods and Processes. In recent years, content platform monitoring and prediction of pandemic diseases through search engines and social networks have become a hot research area. Most of the research is based on search data from Twitter [54] and Google [55], but few are conducted through Baidu and Sina Weibo. This
TABle 7: Data with low BSI period.

\begin{tabular}{lcccc}
\hline Data & Real value & Fitted value & Deviation (\%) & Symbol \\
\hline $2 / 8$ & 38461 & 56576 & 47.10 & - \\
$2 / 14$ & 39332 & 44515 & 13.18 & - \\
$3 / 16$ & 14468 & 15918 & 10.02 & - \\
\hline
\end{tabular}

research integrates multidisciplinary knowledge, uses the SRAF, searches keywords through Weibo to obtain positive emotion data, and uses BSI to obtain risk perception data. Compared with the questionnaire survey, the number of survey target groups is larger, and the scope is wider. The research target can cover the entire survey area more scientifically, and the data are more convincing. Secondly, during the pandemic period, questionnaires, interviews, surveys, and psychological tests are restricted by isolation, martial law, and other situations. Sina Weibo and Baidu hot word search engines provide efficient and scientific research methods. In terms of data representation, during the data collection process of this study, the research object received an emotional statistical survey without their awareness. The information collected is more accurate and credible, and it can better reflect the true feelings of the respondents. It will not be affected by the public subjective level, so the data quality is higher. Similarly, Tao et al. used the comments and search data in social media to analyze the relationship in tourist satisfaction and air quality in the scenic area, providing a reference for scenic area management [56].

5.2. Discussion of Research Results. According to the research results, we believe that the proportion of positive emotions in Weibo is negatively correlated with the BSI, which means that when the public's emotions have a negative tendency, risk perception will be greatly increased. A study proved this conclusion from another angle. Research on employees in Shanghai, China, shows that employees who are more sensitive to the COVID-19 risks show higher negative emotions and work stress [57]; the severity of the pandemic is negatively correlated with the proportion of positive emotions, which means as the severity of the pandemic continues to increase, the people's emotions become negative, and the proportion of positive emotions will show a downward trend. This is not surprising at all. The results of a survey of more than 50,000 people proved our judgment. During the pandemic, the psychological pressure and the sense of frustration of the public were significantly higher [58]; the severity of the pandemic is positively correlated with the BSI, which shows that the increase in the pandemic will cause widespread public concern. People's perception of risk has improved significantly. Information about the deterioration or rapid spread of the pandemic on the Internet may cause the public to panic. The specific manifestation is that the public opinion in Weibo has become pessimistic, and the real value of the positive emotion ratio is much lower than the fitting value of the prediction model; apart from this, the severe pandemic situation will also cause extensive searches by netizens. The real value of the BSI will be much higher than the fitted value of the prediction model, 
indicating that the public's perception of risks has increased significantly. Similar to our research results, Carmen studied the comprehensive indicators of the impact of COVID-19 on the health of community. The research shows that the frequency of online search is highly correlated with the severity of the pandemic [59]. It is interesting and gratifying to find that the strong statements and behaviors of the country or government, and the news events, where the pandemic is controlled or weakened, will cause the public to generate positive emotions, which is specifically expressed by publishing more positive and optimistic Weibo views. The real value of the proportion of positive emotional posts in Weibo is significantly higher than the fitted value of the prediction model. The positive actions of the government and major scientific research progress have reduced people's perception of risk, which is manifested by the reduction in the frequency of online search. The real value of the BSI is significantly lower than the fitted value of the prediction model.

From the statistical graphs drawn by the real values of the three, it can be seen that the changing trend generally conforms to the research conclusion, but in some periods, it does not change completely according to this law, and there are some abnormal mismatches (for example, in some periods, with the increase of the number of newly diagnosed people, the proportion of positive emotions also increases). In this study, the BSI and the proportion of positive emotional posts in Weibo were analyzed. Analyzed by ARIMA time series, the periods with obvious differences were sorted out. After analyzing the important pandemic information and prevention and control policies at home and abroad during these periods, it was found that dramatic changes in the pandemic situation and the prevention and control policies that caused the fluctuation of public's positive emotions and risk perception. This proves the influence of authoritative media on public's emotion and risk perception from the side, and it provides a theoretical reference for government departments to guide the public to correctly view and respond to the pandemic.

\subsection{Lead the Public to Maintain Positive Emotions and} Reasonable Risk Perception. In recent years, the influence of social networks on people's opinions and decisions has become greater and greater, and it has attracted widespread attention [60]. The description of disasters in the network can weaken or enhance the perception of risk and ultimately affect the action [61]. When a crisis or conflict occurs, scientific and effective risk communication is essential. Research results in the field of food safety show that the government uses social media for risk communication, which increases people's positive emotions about food safety and promotes rational risk response [62]. In order to understand how disaster risk is perceived and shared and its impact on public's emotions, the SRAF provides a perspective for observing risks. Different communication media and amplification stations can affect public risk perception [63]. Studies have shown that nonprofessional groups are more sensitive to public hazards than experts. Nonauthoritative groups, as information amplifying stations, may transform information and increase social risk perception [64]. At a time when global connections are getting closer, the Internet has become more open, making it possible for false and harmful information to spread through social media. So far, some people have strictly adhered to these restrictions to prevent infection, such as maintaining hand hygiene and necessary social isolation. However, others have ignored or procrastinated government regulations and still frequently appear on crowded beaches or bar [65]. This eloquently proves that the public's emotions and perception of risks associated with COVID-19 differ greatly in different places and individuals [15].

The government, relying on official media such as television, newspapers, and the Internet, plays an important role in notifying the public about health issues, shaping the public's attitude toward pandemic policies, and influencing public decision-making and behavior [66]. The government establishes a clear and coordinated communication strategy to deal with major public health disasters, which can gain the trust of citizens in a rapidly developing situation and avoid the public's blind optimism or panic about the pandemic [1]. The government's words and deeds on social media can influence the individual's antiepidemic actions. Correspondingly, the government and policy makers are also inspired by the public's emotions and cognition in online media [7]. Enhancing accurate and adequate information communication between the government and the public can help the public maintain positive emotions and establish a good sense of risk. In the early stage of the epidemic, the Vietnamese government used video and music on the Internet to guide people to form healthy habits and achieved good results [67]. The research by Chen and Davies proves this and believes that the government maintains an open way to accurately and strategically explain the health crisis facing the public, which helps reduce public anger and panic. $[16,53]$. Obviously, doing this job well is a challenge for every country and government. This research has an important reference value for leaders and decision makers to deal with similar disasters and crises. Because it provides the connection and influencing factors of the public's positive emotions and risk perceptions in crises situations, it proves that the information in social media will greatly affect the public's cognition and behavior. This knowledge once again reminds the government to pay attention to the role of social media in disaster management, enhance the communication between the government and the public, and guide the public to maintain positive emotions and reasonable risk perception.

5.4. Limitations and Future Directions. This study has several limitations. Firstly, this study focuses on the dynamic changes of the public's positive emotions and risk perception in Hubei Province during the pandemic. Therefore, the sample source is limited to Hubei, and the sample range can be expanded in subsequent studies. Secondly, online data collection and analysis did not include all the public in the research scope. Individuals such as the elderly and children who are not good at online communication cannot be 
investigated. Because the network is virtual, it cannot accurately reflect the impact of gender and age differences on public's emotion and risk perception during the pandemic. Finally, the judgment of positive emotions in this study mainly relies on the text of Weibo comments, expressions, and image expressions in Weibo communication [27], which are not included in the statistics due to the limitation of emotion division standards. In the follow-up research, these limitations deserve careful analysis and discussion.

\section{Conclusion}

This study collects data related to COVID-19 in Hubei Province, China, from January 20 to April 8, 2020, including the proportion of positive emotional posts in Weibo, the number of newly diagnosed daily, and the BSI. Through analysis and research on the correlation, linear regression, and ARIMA time-series model of the three sets of data, the following conclusions are drawn. The proportion of positive emotional posts in Weibo is negatively correlated with the level of risk perception, the severity of the pandemic is negatively correlated with the proportion of positive emotions, and the severity of the pandemic is positively correlated with the degree of risk perception. Information about the deterioration or rapid spread of the pandemic on the Internet may cause the public to panic. Specifically, the real value of the positive emotion ratio of Weibo is significantly lower than the fitting value of the prediction model, the number and frequency of Baidu searches increase, and the public's level of risk perception is significantly improved. Contrary to this, the strong decision-making, behavior, and positive pandemic news events of the state or government will cause the public to generate positive emotions, reduce the number and frequency of Baidu searches, and reduce people's risk perception level. By observing the changing trend of variables, the proportion of positive emotional posts in Weibo, the number of newly diagnosed cases, and the BSI, it can be found that the volatility of the curve generally conforms to the relationship drawn from the research conclusions. However, at certain specific points in time, sudden pandemic events or government decisions can cause some abnormal fluctuations in the proportion of positive emotions and BSI. Based on the research results, we outline some policy suggestions.

The public is increasingly using social media to gain the risk perception of the pandemic and express their emotions. Therefore, the government must have the necessary tools to extract this valuable information in a comprehensive manner and consider this in the subsequent decisionmaking process.

The words and deeds of the government greatly affect the decision-making and behavior of the public. The government's communication of risk information with the public in official media such as television, newspapers, and the Internet can guide the public to maintain the positive emotions and reasonable risk perception. This is of great significance in this long-term struggle against COVID-19.

\section{Data Availability}

The original data used to support the findings of this study are available from the corresponding author upon request.

\section{Conflicts of Interest}

The authors declare that there are no conflicts of interest regarding the publication of this paper.

\section{Acknowledgments}

This work was supported by the MOE (Ministry of Education in China) Project of Humanities and Social Sciences (18YJCZH191) and Fundamental Research Funds for the Central Universities of China (2652019073).

\section{References}

[1] Y. B. D. Bruin, A.-S. Lequarre, J. McCourt et al., "Initial impacts of global risk mitigation measures taken during the combatting of the COVID-19 pandemic," Safety Science, vol. 128, Article ID 104773, 2020.

[2] R. M. Anderson, H. Heesterbeek, D. Klinkenberg, and T. D. Hollingsworth, "How will country-based mitigation measures influence the course of the COVID-19 epidemic?" The Lancet, vol. 395, no. 10228, pp. 931-934, 2020.

[3] E. A. Waters, "Feeling good, feeling bad, and feeling at-risk: a review of incidental affect's influence on likelihood estimates of health hazards and life events," Journal of Risk Research, vol. 11, no. 5, pp. 569-595, 2008.

[4] Y. Liu, C. Xie, S. She et al., "Emotion and aversion towards timing ambiguity in the perception of environmental risks," in Proceedings of the 2013 International Conference on Management Science and Engineering (ICMSE), pp. 1433-1438, Harbin, China, July 2013.

[5] P. Slovic, "Perception of risk," Science, vol. 17, pp. 43-54, 1987.

[6] R. N. Rimal and K. Real, "Perceived risk and efficacy beliefs as motivators of change: use of the risk perception attitude (RPA) framework to understand health behaviors," Human Communication Research, vol. 29, no. 3, p. 370, 2003.

[7] S. T. Heydari, L. Zarei, A. K. Sadati et al., "The effect of risk communication on preventive and protective behaviours during the COVID-19 outbreak: mediating role of risk perception," BMC Public Health, vol. 21, no. 1, 2021.

[8] D. Herrero-Fernández, P. Parada-Fernández, M. OlivaMacías, and R. Jorge, "The influence of emotional state on risk perception in pedestrians: a psychophysiological approach," Safety Science, vol. 130, Article ID 104857, 2020.

[9] R. M. Hogarth, M. Portell, and A. Cuxart, "What risks do people perceive in everyday life? A perspective gained from the experience sampling method (ESM)," Risk Analysis, vol. 27, no. 6, pp. 1427-1439, 2007.

[10] A. Bec and S. Becken, "Risk perceptions and emotional stability in response to Cyclone Debbie: an analysis of twitter data," Journal of Risk Research, Article ID 1673798, 2019.

[11] E. Samadipour, F. Ghardashi, and N. Aghaei, "Evaluation of risk perception of COVID-19 disease: a community-based participatory study," Disaster Medicine and Public Health Preparedness, vol. 2, pp. 1-20, 2020. 
[12] J. Dyer and B. Kolic, "Public risk perception and emotion on Twitter during the Covid-19 pandemic," Applied Network Science, vol. 5, no. 1, p. 99, 2020.

[13] Q. Yan, Y. Tang, D. Yan et al., "Impact of media reports on the early spread of COVID-19 epidemic," Journal of Theoretical Biology, vol. 502, Article ID 110385, 2020.

[14] M. A. Borg, "Cultural determinants of infection control behaviour: understanding drivers and implementing effective change," Journal of Hospital Infection, vol. 86, no. 3, pp. 161-168, 2014.

[15] T. L. D. Huynh, "Does culture matter social distancing under the COVID-19 pandemic?" Safety Science, vol. 130, Article ID 104872, 2020.

[16] Q. Chen, C. Min, W. Zhang, G. Wang, X. Ma, and R. Evans, "Unpacking the black box: how to promote citizen engagement through government social media during the COVID19 crisis," Computers in Human Behavior, vol. 110, Article ID 106380, 2020

[17] W. Huang, X. Yao, and Q. Wang, "Research on Weibo emotion classification based on context," Lecture Notes in Computer Science (Including Subseries Lecture Notes in Artificial Intelligence and Lecture Notes in Bioinformatics), vol. 11354, pp. 222-231, 2019.

[18] K. Liu, T. Wang, Z. Yang et al., "Using Baidu Search Index to predict dengue outbreak in China," Scientific Reports, vol. 6, p. 38040, 2016.

[19] N. Xu and X. Tang, "A causality analysis of societal risk perception and stock Market volatility in China," Journal of Systems Science and Systems Engineering, vol. 27, no. 5, pp. 613-631, 2018.

[20] B. L. Fredrickson, "The role of positive emotions in positive psychology: the broaden-and-build theory of positive emotions," American Psychologist, vol. 56, no. 3, pp. 218-226, 2001.

[21] R. Reisenzein, "Cognition and emotion: a plea for theory," Cognition and Emotion, vol. 33, no. 1, pp. 109-118, 2019.

[22] B. Pang and L. Lee, "Opinion mining and sentiment analysis," Foundations and Trends in Information Retrieval, vol. 2, no. 12, pp. 1-135, 2008.

[23] A. Hartanto, J. C. Yong, W. X. Toh, S. T. H. Lee, G. Y. Q. Tng, and W. Tov, "Cognitive, social, emotional, and subjective health benefits of computer use in adults: a 9-year longitudinal study from the Midlife in the United States (MIDUS)," Computers in Human Behavior, vol. 104, Article ID 106179, 2020.

[24] A. Pepe and J. Bollen, "Between conjecture and memento: shaping a collective emotional perception of the future," 2008, https://arxiv.org/abs/0801.3864.

[25] Y. Dong, H. Chen, X. Tang et al., "Collective emotional reaction to societal risks in China," in Proceedings of the 2015 IEEE International Conference on Systems, Man, and Cybernetics, IEEE, Hong Kong, China, October 2015.

[26] D. Watson and A. Tellegen, "Toward a consensual structure of mood," Psychological Bulletin, vol. 98, no. 2, pp. 219-235, 1985.

[27] F. Jiang, Y.-Q. Liu, H.-B. Luan et al., "Microblog sentiment analysis with emoticon space model," Journal of Computer Science and Technology, vol. 30, no. 5, pp. 1120-1129, 2015.

[28] S. Mashwani, "The coronavirus (COVID-19) pandemic's impact on mental health," International Journal of Health Planning and Management, vol. 35, no. 5, pp. 993-996, 2020.

[29] C. Starr, "Social benefit versus technological risk," Science, vol. 165, no. 3899, pp. 1232-1238, 1969.

[30] B. Fischhoff, P. Slovic, S. Lichtenstein, S. Read, and B. Combs, "How safe is safe enough? A psychometric study of attitudes towards technological risks and benefits," Policy Sciences, vol. 9, no. 2, pp. 127-152, 1978.

[31] P. Slovic, B. Fischhoff, and S. Lichtenstein, "Why study risk perception?” Risk Analysis, vol. 2, no. 2, pp. 83-93, 1982.

[32] G. Weinstein and M. Douglas, "Risk and culture: an essay on the selection of technological and environmental dangers acceptable risk," American Political Science Review, vol. 77, 1983.

[33] A. L. PedrosaL. Bitencourt et al., "Emotional, behavioral, and psychological impact of the COVID-19 pandemic," Frontiers in Psychology, vol. 11, Article ID 566212, 2020.

[34] S. Dryhurst, C. R. Schneider, J. Kerr et al., "Risk perceptions of COVID-19 around the world," Journal of Risk Research, vol. 23, no. 7-8, pp. 994-1006, 2020.

[35] N. C. Bronfman and L. A. Cifuentes, "Risk perception in a developing country: the case of Chile," Risk Analysis, vol. 23, no. 6, pp. 1271-1285, 2003.

[36] A. R. Roberts, "Assessment, crisis intervention, and trauma treatment: the integrative ACT intervention model," Brief Treatment and Crisis Intervention, vol. 2, no. 1, pp. 1-22, 2002.

[37] J. C. Coyne and G. Downey, "Social factors and psychopathology: stress, social support, and coping processes," Annual Review of Psychology, vol. 42, no. 1, pp. 401-425, 1991.

[38] X. Tang, "Applying search words and BBS posts to societal risk perception and harmonious society measurement," in Proceedings of the 2013 IEEE International Conference on Systems, Man, and Cybernetics (SMC 2013), pp. 2191-2196, Manchester, UK., October 2013.

[39] R. E. Kasperson, O. Renn, P. Slovic et al., "The social amplification of risk: a conceptual framework," Risk Analysis, vol. 8, no. 2, pp. 177-187, 1988.

[40] M. K. Lindell and R. W. Perry, "The protective action decision model: theoretical modifications and additional evidence," Risk Analysis, vol. 32, no. 4, p. 616, 2012.

[41] M. K. Lindell and S. N. Hwang, "Households' perceived personal risk and responses in a multihazard environment," Risk Analysis, vol. 28, no. 2, pp. 539-556, 2008.

[42] S. M. Pandit and S.-M. Wu, Time Series and System Analysis, with Applications, Wiley, Hoboken, NJ, USA, 1983.

[43] X. Li, C. Zhang, B. Zhang, and K. Liu, "A comparative time series analysis and modeling of aerosols in the contiguous United States and China," Science of the Total Environment, vol. 690, pp. 799-811, 2019.

[44] M. Perzyk, K. Krawiec, and J. Kozłowski, "Application of time-series analysis in foundry production," Archives of Foundry Engineering, vol. 9, no. 3, pp. 109-114, 2009.

[45] P. Narayanan, A. Basistha, S. Sarkar, and S. Kamna, "Trend analysis and ARIMA modelling of pre-monsoon rainfall data for western India," Comptes Rendus Geoscience, vol. 345, no. 1, pp. 22-27, 2013.

[46] S. Singh, K. S. Parmar, J. Kumar et al., ““'Development of new hybrid model of discrete wavelet decomposition and autoregressive integrated moving average (ARIMA) models in application to one month forecast the casualties cases of COVID-19," Chaos," Solitons \& Fractals, vol. 135, 2020.

[47] L. Zhou, J.-M. Liu, and X.-P. Dong, "COVID-19 seeding time and doubling time model: an early epidemic risk assessment tool," Infectious Diseases of Poverty, vol. 9, no. 1, pp. 1-9, 2020.

[48] D.-M. Xue and Z.-Q. Hua, "ARIMA based time series forecasting model," Recent Advances in Electrical \& Electronic Engineering (Formerly Recent Patents on Electrical \& Electronic Engineering), vol. 9, no. 2, pp. 93-98, 2016.

[49] M. N. Martinez and M. J. Bartholomew, "What does it "mean"? A review of interpreting and calculating different 
types of means and standard deviations," Pharmaceutics, vol. 9, no. 2, 2017.

[50] S. Bhandari and M. Hallowell, Emotional States and Their Impact on Hazard Identification Skills, ASCE Library, Reston, VA, USA, 2016.

[51] D.-H. Choi, W. Yoo, G.-Y. Noh, and K. Park, "The impact of social media on risk perceptions during the MERS outbreak in South Korea," Computers in Human Behavior, vol. 72, pp. 422-431, 2017.

[52] T. Duan, H. Jiang, and X. Deng, "Government intervention, risk perception, and the adoption of protective action recommendations: evidence from the COVID-19 prevention and control experience of China," International Journal of Environmental Research and Public Health, vol. 17, no. 10, p. E3387, 2020.

[53] G. Davies, "The epidemic severity index: estimating relative local severity of novel disease outbreaks," 2020.

[54] P. Panagiotopoulos, J. Barnett, A. Z. Bigdeli, and S. Sams, "Social media in emergency management: twitter as a tool for communicating risks to the public," Technological Forecasting and Social Change, vol. 111, pp. 86-96, 2016.

[55] J. R. Ortiz, H. Zhou, D. K. Shay et al., "Monitoring influenza activity in the United States: a comparison of traditional surveillance systems with Google Flu Trends," PLoS One, vol. 6, no. 4, p. e18687, 2011.

[56] Y. Tao, F. Zhang, C. Shi et al., "Social media data-based sentiment analysis of tourists' air quality perceptions," Sustainability (Switzerland), vol. 1118 pages, 2019.

[57] C. A. Warden, A. R. Warden, S. C.-T. Huang et al., "Job tension and emotional sensitivity to COVID-19 public messaging and risk perception," Population Health Management, 2020.

[58] Q. Han, B. Zheng, M. Agostini et al., "Associations of risk perception of COVID-19 with emotion and mental health during the pandemic," Journal of Affective Disorders, vol. 284, no. 1, pp. 247-255, 2021.

[59] C. Herrero and A. Villar, "A synthetic indicator on the impact of COVID-19 on the community's health," PLoS One, vol. 15, no. 9, Article ID e0238970, 2020.

[60] W. Li and H. Xu, "Text-based emotion classification using emotion cause extraction," Expert Systems with Applications, vol. 41, no. 4, pp. 1742-1749, 2014.

[61] N. Pidgeon and K. Henwood, "Chapter 4. the social amplification of risk framework (SARF): theory, critiques, and policy implications," Risk Communication and Public Health, pp. 53-69, 2010.

[62] T. M. Ha, S. Shakur, and K. H. Pham Do, "Linkages among food safety risk perception, trust and information: evidence from Hanoi consumers," Food Control, vol. 110, 2020.

[63] D. E. Alexander, "Social media in disaster risk reduction and crisis management," Science and Engineering Ethics, vol. 20, no. 3, pp. 717-733, 2014.

[64] J. Fellenor, J. Barnett, C. Potter, J. Urquhart, J. D. Mumford, and C. P. Quine, "The social amplification of risk on twitter: the case of ash dieback disease in the United Kingdom," Journal of Risk Research, vol. 21, no. 10, pp. 1163-1183, 2018.

[65] L. Cori, F. Bianchi, E. Cadum et al., "Risk perception and COVID-19," International Journal of Environmental Research and Public Health, vol. 17, no. 9, 2020.

[66] T. A. Morton and J. M. Duck, "Communication and health beliefs," Communication Research, vol. 28, no. 5, pp. 602-626, 2001.

[67] H. T. L. Duc, "The COVID-19 containment in Vietnam: what are we doing?" Journal of Global Health, vol. 10, no. 1, 2020. 